\title{
Modo de uso dos medicamentos de portadores de doenças crônicas acompanhados pelas equipes da estratégia de saúde da família de um município mineiro
}

\author{
Medication use mode of people with chronic diseases accompanied \\ by the work teams of the family health strategy from a municipality \\ of Minas Gerais, Brazil
}

Nádia Camila Rodrigues Costa Caixeta, ${ }^{1}$ Elias de Sousa Tonelini, ${ }^{2}$ Douglas Cardoso Brandão, ${ }^{3}$

Ricardo Ferreira Nunes, ${ }^{3}$ Salvador Boccaletti Ramos, ${ }^{1}$ Glória Lúcia Alves Figueiredo

Universidade de Franca (UNIFRAN), Franca, SP, Brasil.

2Centro Universitário de Patos de Minas (UNIPAM), Patos de Minas, MG, Brasil.

«universidade de Brasilia (UNB), Brasilia, DF, Brasil.

Recebido em: 21/08/2016 / Aceito em: 26/09/2016 / Publicado em: 18/10/2016

nadiac.caixeta@gmail.com

\section{RESUMO}

Objetivo: avaliar o modo de uso dos medicamentos prescritos aos portadores de doenças crônicas não transmissíveis (DCNT), atendidos pelas equipes da Estratégia de Saúde da Família (EqSF), de um município mineiro. Método: trata-se de um estudo descritivo, de corte transversal, realizado com 34 famílias (52 indivíduos com DCNT). Os dados foram coletados por meio de entrevista domiciliar, através do aplicativo droidSURVEY, disponibilizado em tablets e dispositivos móveis de smartphones para armazenamento dos dados. Para a análise estatística utilizou-se o software $R$, versão 3.3.0. Resultados: nas visitas domiciliares, resultaram em 52 indivíduos portadores de DCNT, sendo 37\% considerados não aderentes ao tratamento medicamentoso devido ao desacordo posológico. Desses, 19\% usavam a medicação com a dose diferente da receita médica, $59 \%$ faziam uso dos medicamentos em horários distintos do prescrito, $22 \%$ relataram falta de entendimento da prescrição médica. Considerações finais: o modo de uso dos medicamentos prescritos foi avaliado como inadequado, assim, sugere-se às EqSF analisar as dificuldades encontradas e propor estratégias mais efetivas de incentivo à adesão ao tratamento. No entanto, o envolvimento entre a EqSF e os familiares no cuidado aos indivíduos portadores de DCNT é essencial para manutenção sistemática da terapia medicamentosa.

Palavras-chave: Uso de Medicamentos; Doença crônica; Estratégia de Saúde da Família.

\section{ABSTRACT}

Objective: to assess the use mode of the prescribed drugs to patients with chronic non-communicable diseases (CNCDs), assisted by the Family Health Strategy (EqSF) work teams from a municipality of Minas Gerais, Brazil. Method: this is a descriptive and cross-sectional study, held with 34 families 152 individuals with CNCDs). Data were collected through home interviews, through droidSURVEY application, available on tablets and mobile phones for data storage devices. Statistical analysis was performed using the $R$ software, version 3.3.0. Results: the home visits resulted in 52 individuals with CNCDs, in which $37 \%$ were considered non-adherent to the medication treatment due to the dose disagreement. $19 \%$ of these used the medication with a different dosage from the prescription; 59\% were using the medications at different times of the prescribed ones; $22 \%$ reported misunderstanding the prescription. Concluding remarks: the using mode of prescribed drugs was evaluated as inappropriate, therefore, it is suggested to the Family Health Strategy work teams (EqSF) to analyses the difficulties detected and propose more effective strategies encouraging the treatment compliance. However, the engagement between the EqSF and the family members when it comes to the people with CNCDs care is crucial for the drug therapy systematic maintenance.

Keywords: Drug utilization; Chronic disease; Family Health Strategy. 


\section{INTRODUÇÃO}

No Brasil, as Doenças Crônicas Não Transmissíveis (DCNT) constituem o problema de saúde de maior magnitude, associado ao envelhecimento da população. Correspondem a $72 \%$ das causas de mortes, atingindo fortemente camadas pobres da população e grupos vulneráveis, principalmente os idosos. ${ }^{1}$

Após o diagnóstico da doença crônica, é indispensável a adesão ao tratamento, pois o não seguimento do plano terapêutico poderá repercutir de forma negativa sobre a saúde das pessoas acometidas por essas enfermidades, impossibilitando a resolubilidade do tratamento. $^{2}$

A não adesão ao tratamento é considerada um fenômeno complexo e multifatorial, estando relacionados ao paciente, ao sistema e equipe de saúde, à doença, ao tratamento, ao uso de medicamentos, a problemas sociais e a fatores socioeconômicos. ${ }^{2}$

Estimativas indicam que o grau de não adesão mundial aos tratamentos de doenças crônicas varia de $30 \%$ a $50 \%$, sendo mais evidente no tratamento medicamentoso, tendo como fator desencadeador o número de medicamentos consumidos. ${ }^{3}$

No âmbito da Atenção Primária da Saúde (APS) nacional e da estratégia de saúde da família (ESF), faz-se necessária a orientação ao indivíduo portador de DCNT quanto ao uso correto dos medicamentos, escoIha do local adequado e seguro para armazenamento e conferência da data de validade. ${ }^{4}$

Estudiosos defendem que o insucesso da farmacoterapia e o controle das doenças crônicas podem estar relacionados principalmente ao medicamento. $\mathrm{Na}$ pesquisa realizada por Mastroianni et al., ${ }^{4}$ verificou-se a presença de medicamentos em 91,1\% dos domicílios visitados. Desses, $75,8 \%$ eram armazenados em locais inadequados e 7,5\% usavam o medicamento de forma diferente da receita médica.

As visitas domiciliares preconizadas para as EqSF se caracterizam como uma atividade externa à unidade de saúde com o objetivo de conhecer e avaliar as necessidades das famílias cadastradas e do ambiente onde vivem. Essas informações são fundamentais para se conhecer melhor o ambiente familiar, facilitar a identificação de problemas relacionados, inclusive quanto aos medicamentos e prevenção dos agravos das DCNT.5,6 Nesse cenário em que se identificam as principais barreiras associadas ao uso inadequado dos medicamentos, caberia às EqSF promover a adesão ao tratamento, de modo a garantir aos pacientes uma compreensão da necessidade do tratamento para o controle da DCNT. ${ }^{2}$

Percebe-se um interesse crescente pela prevenção e controle das DCNT e de seus fatores de risco. Assim, acredita-se que o conhecimento das experiências familiares com a medicação possa dar visibilidade as fragilidade e fortalezas desse cuidado, a fim de fornecer subsídios para os serviços de saúde públicos implementarem ações na perspectiva da promoção em saúde.

Diante deste contexto, o objetivo deste trabalho foi avaliar o modo de uso dos medicamentos prescritos aos portadores de DCNT, atendidos pelas equipes da Estratégia de Saúde da Família (EqSF) de um município mineiro.

\section{MÉTODO}

Trata-se de um estudo descritivo, de corte transversal, que buscou avaliar o modo de uso dos medicamentos prescritos aos portadores de DCNT, atendidos pelas EqSF de um município mineiro. Essa pesquisa atendeu às recomendações da resolução do Conselho Nacional de Saúde 466/2012, obtendo autorização do CEPE da Universidade de Franca - UNIFRAN (número de parecer: 1.250.044).

O campo da pesquisa foi o município de Presidente Olegário, no interior de Minas Gerais, que se encontra a $423 \mathrm{Km}$ da capital mineira, Belo Horizonte e conta com uma população de aproximadamente 18.577 habitantes. ${ }^{7} \mathrm{O}$ cenário da pesquisa foram os domicílios de indivíduos portadores de DCNT, cadastrados pelas unidades da ESF do município citado, que abrange seis equipes de Saúde da Família.

A amostra foi definida utilizando-se o cálculo de amostragem de proporção, com intervalo de confiança de 95\% (IC 95\%), totalizando 34 famílias. A escolha dos domićlios foi de modo aleatório e estratificado, considerando as seis microáreas da ESF. Adotou-se como critério de inclusão, participantes moradores nos domicílios escolhidos, com idade acima de dezoito anos para responder o questionário e que aceitaram representar a família e assinar o Termo de Consentimento Livre e Esclarecido (TCLE). Foram excluídos aqueles moradores que não atenderam os entrevistadores em até três tentativas.

Após autorização das Secretarias Municipais de Saúde e coordenadores das unidades de saúde e antes do início da coleta de dados, a pesquisadora apresentou o projeto aos profissionais das EqSF com a finalidade de esclarecer sobre o conteúdo do mesmo e solicitar a colaboração dos profissionais.

Após a identificação dos domicílios, a pesquisadora, juntamente aos ACS visitaram as famílias para as entrevistas. Aqueles que aceitaram participar da pesquisa, assinaram o TCLE para iniciar a coleta de dados. E, para assegurar aos participantes o sigilo e anonimato, cada família foi identificada com as inicias do nome do município (PO - Presidente Olegário) e o número do prontuário da família. Em caso de repetição do número do prontuário, acrescentou-se uma letra do alfabeto, na sequência.

O instrumento para coleta de dados foi um questionário composto de questões fechadas, o qual foi importado para o aplicativo droidSURVEY com a finalidade de coletar os dados usando tablet ou dispositivos móveis de smartphones, para evitar erros com a digitação dos dados. Esse software não precisa de conexão com a internet enquanto está realizando a pesquisa. Os dados foram armazenados de forma segura no tablet ou dispositivos móveis de smartphones, em seguida, com conexão à internet, os dados foram enviados automaticamente ou por transferência manual para o website e possibilitou a visualização e análise dos dados pelo computador

$\mathrm{Na}$ visita domiciliar, foi solicitado aos indivíduos portadores de DCNT que mostrassem os medicamentos em uso para tratamento das DCNT e as receitas médicas, prescritas pelo médico da EqSF. Cada indivíduo foi questionado quanto ao modo de uso de cada medi- 
camento e o entrevistador verificava na última receita médica (com data de emissão inferior a seis meses) o nome do medicamento, dosagem e modo de uso; para conferência do relato e posterior classificação.

O modo de uso dos medicamentos prescritos foi classificado segundo Mastroianni et al. ${ }^{4}$ Considerou-se o modo de uso adequado, aquele em que o conjunto de medicamentos declarado pelo indivíduo fosse o mesmo prescrito na receita médica. Já para o modo de uso inadequado dos medicamentos foram considerados aqueles que estavam em desacordo posológico (ou seja, quando a frequência ou dose mencionada pelo morador estivessem diferentes da receita médica) e aqueles em que houve o relato da interrupção do tratamento.

Para classificação do modo de uso inadequado, o entrevistador registrou a situação encontrada no momento da entrevista, tais como: dosagem errada, horário incorreto, troca de medicação por opção, não toma todos os medicamentos prescritos, não entendeu como tomar, não tem ajuda de um familiar, fica o dia todo sozinho, não quer tomar a medicação, não acredita no tratamento, não aceita o diagnóstico, só toma quando está em crise, não tem o medicamento ou outro.

$\mathrm{Na}$ análise estatística, as variáveis categóricas foram descritas por meio de frequências absoluta (N) e relativa (\%). As associações entre a variável 'classificação do modo de uso dos medicamentos' e as variáveis socioeconômicas foram estimadas por meio do teste Qui-Quadrado considerando um nível de significância igual a 0.05. A comparação entre a proporção de pessoas que tomam todos os medicamentos seguindo a prescrição médica e a proporção de pessoas que tomam ao menos um medicamento fora das prescrições foi feita por meio do teste binomial com nível de significância de 0.05 (5\%). Todas as análises foram feitas utilizando o software $R$, versão 3.3.0.8

\section{RESULTADOS E DISCUSSÃO}

As visitas domiciliares foram realizadas em 34 residências, resultando em 52 indivíduos portadores de DCNT atendidos pela EqSF e que faziam uso de medicamento para tratamento das doenças crônicas. Os dados da pesquisa caracterizaram-se por 29 mulheres (56\%), 31 casados (60\%), 21 idosos com idade entre 70 e 79 anos (40\%), 46 relataram ter baixa escolaridade (46\%), além de 44 apresentarem baixa renda salarial (85\%), sendo que essas variáveis não obtiveram significância estatística por meio do teste Qui-Quadrado $(p>0,05)$.

As DCNT predominantes nos participantes foram hipertensão arterial sistêmica (77\%), diabetes mellitus (25\%), doença mental (15\%) e hipotireoidismo (12\%), apresentando co-morbidades em vários indivíduos.

Com base no teste comparativo binomial, dos 52 indivíduos portadores de DCNT, 19 foram considerados não aderentes ao tratamento medicamentoso, representando uma prevalência de não adesão de $37 \%$. Sendo o $p>0.05$ não houve diferença estatística significativa entre as proporções de indivíduos que seguiram a prescrição médica corretamente e aqueles que cometeram algum erro na tomada do medicamento prescrito.

Segundo a Organização Mundial de Saúde (OMS), ${ }^{9}$ o conceito de adesão pode ser definido como "o
Tabela 1 - Distribuição das características socioeconômicas e prevalência da adesão ao tratamento de portadores de DCNT's atendidos pela Estratégia de Saúde da Família de Presidente Olegário, MG.

\begin{tabular}{|c|c|c|c|c|c|}
\hline \multirow[b]{3}{*}{ Características } & \multicolumn{4}{|c|}{ Adesão } & \multirow[b]{3}{*}{$P$} \\
\hline & \multicolumn{2}{|c|}{$\operatorname{Sim}(n=33)$} & \multicolumn{2}{|c|}{ Não $(n=19)$} & \\
\hline & $\mathrm{n}$ & $\%$ & $\mathrm{n}$ & $\%$ & \\
\hline \multicolumn{6}{|l|}{ Sexo } \\
\hline Homens & 13 & 25 & 10 & 19 & 0.525 \\
\hline Mulheres & 20 & 38 & 9 & 17 & \\
\hline \multicolumn{6}{|l|}{ Estado civil } \\
\hline Casados & 18 & 35 & 13 & 25 & 0.288 \\
\hline Viúvos & 9 & 17 & 2 & 4 & \\
\hline Divorciados & 4 & 8 & 1 & 2 & \\
\hline Solteiros & 2 & 4 & 3 & 6 & \\
\hline \multicolumn{6}{|l|}{ Idade } \\
\hline $40-49$ anos & 1 & 2 & 4 & 8 & 0.3582 \\
\hline $50-59$ anos & 5 & 10 & 3 & 6 & \\
\hline $60-69$ anos & 6 & 12 & 4 & 8 & \\
\hline $70-79$ anos & 14 & 27 & 7 & 14 & \\
\hline Mais de 80 anos & 7 & 13 & 1 & 2 & \\
\hline \multicolumn{6}{|l|}{ Escolaridade } \\
\hline Não alfabetizado & 7 & 13 & 4 & 8 & 0.2384 \\
\hline Menos de 4 anos & 18 & 35 & 6 & 12 & \\
\hline Entre 4 a 7 anos & 8 & 15 & 7 & 13 & \\
\hline Entre 8 a 10 anos & 0 & 0 & 1 & 2 & \\
\hline Mais de 11 anos & 0 & 0 & 1 & 2 & \\
\hline \multicolumn{6}{|l|}{ Renda salarial } \\
\hline Sem rendimento & 1 & 2 & 1 & 2 & 0.6911 \\
\hline Menos de 1 salário mínimo & 3 & 6 & 3 & 6 & \\
\hline Entre 1 e 3 salários mínimos & 29 & 56 & 15 & 29 & \\
\hline
\end{tabular}

comportamento de uma pessoa em relação ao uso de medicamentos, seguimento da dieta ou mudanças de estilo de vida correspondente às recomendações de um profissional da saúde".

Existem vários métodos para avaliação da adesão ao tratamento farmacológico das DCNT. Os métodos diretos são utilizados para quantificar o fármaco no sangue ou seus metabólitos na urina ou ainda por utilizar um composto traçador associado ao medicamento em uso. Já os métodos indiretos utilizam relato do paciente, avaliação pelo médico, diário do paciente, contagem de comprimidos, reabastecimento de comprimidos, monitorização eletrônica da medicação, resposta clínica e entrevista clínica. ${ }^{10}$

0 método utilizado neste estudo foi o relato do paciente, que consistiu em solicitar ao indivíduo que respondesse algumas perguntas, previamente definidas ao entrevistador. Ele foi utilizado por ser de fácil aplicação e ter a possibilidade de estimar as barreiras de adesão ao tratamento. ${ }^{11}$

A não adesão ao tratamento farmacológico foi classificada em $37 \%$ dos portadores de DCNT em função da situação encontrada durante a entrevista, estando em desacordo com a posologia prescrita aqueles indivíduos que estavam em uso da dose do medicamento diferente da prescrição (19\%) e em horários também distintos do prescrito (59\%). Um fato importante é que $22 \%$ dos indivíduos relataram falta de entendimento da prescrição médica, principalmente quando se referia aos idosos que moravam sozinhos.

$\mathrm{Na}$ literatura levantada, os autores evidenciaram 
que o perfil sócio demográfico, o próprio paciente, o relacionamento do paciente com o profissional de saúde, o serviço de saúde, o tratamento e os medicamentos como sendo as principais causas a interferirem no processo de adesão ao tratamento e aos medicamentos. ${ }^{2,12}$

Quanto ao sexo, observou-se que $17 \%$ das mulheres e $20 \%$ dos homens faziam uso dos medicamentos em desacordo com a prescrição médica. Apesar de ser verificada tendência para a não adesão ao tratamento farmacológico dos homens quando comparado as muIheres, não houve diferença estatisticamente significativa entre os sexos para a não adesão $(p=0.525)$, evidenciando que ambos não aderem ao tratamento. No entanto, historicamente, os homens apresentam maior desconhecimento sobre o seu tratamento, enquanto que as mulheres parecem ter uma percepção mais acurada de sua condição de saúde e desenvolvem maiores relações com o serviço de saúde, principalmente por serem donas de casa e possuírem maior flexibilidade de tempo. ${ }^{13}$

Sobre a variável renda salarial, verificou-se que $4 \%$ dos entrevistados encontravam-se desempregados, $12 \%$ relataram ter renda inferior a um salário mínimo e $85 \%$ possuía condição financeira compreendida entre um e três salários mínimos. Acredita-se que o fator salarial possa ter influenciado a não adesão ao tratamento, devido ao custo de certos medicamentos com indisponibilidade da dose prescrita para acesso gratuito pelo SUS e a falta de adequação da prescrição médica. ${ }^{14,15}$

Referente a escolaridade, $29 \%$ dos entrevistados possuía o tempo de escolaridade entre quatro e sete anos, $46 \%$ relataram ter frequentado menos de quatro anos de estudos e $21 \%$ se diziam analfabetos. No estudo realizado por Barreto et al., ${ }^{3}$ constatou-se que os indivíduos com hipertensão arterial sistêmica (HAS) que possuíam oito anos ou menos de estudo apresentaram chance 1,3 vezes maior de não aderirem a farmacoterapia. Desta forma, sugere-se que a baixa escolaridade possa estar associada a dificuldade em compreender a prescrição médica e a importância do uso contínuo dos medicamentos para o controle das doenças crônicas (DC).

Outra característica evidenciada na população em estudo foi a faixa etária, em que $10 \%$ relataram ter entre 40 e 49 anos, $16 \%$ entre 50 e 59 anos, $20 \%$ entre 60 e 69 anos, $40 \%$ entre 70 e 79 anos e por fim 15\% apresentaram ter mais de 80 anos de idade. Não houve significância estatística ( $p=0.3582)$ entre as idades e ao uso correto dos medicamentos, mostrando que em todas as idades os indivíduos não aderem ao tratamento. Contrapondo o estudo, Barreto et al. ${ }^{3}$ e Busnello et al. ${ }^{16}$ afirmaram que o aumento da idade etária associado à maior probabilidade de seguimento ao tratamento recomendado, no entanto existe um fator associado que são os lapsos da memória nos idosos. O esquecimento pode apontar como causa do uso incorreto dos medicamentos e logo a não adesão ao tratamento. ${ }^{17,18}$

Já os pacientes com idade inferior a 60 anos, apresentaram auto percepção de "boa saúde", se sentem menos vulneráveis e demostraram menor aceitação do tratamento como alternativa de prolongamento da vida. $^{3}$ Devido à ausência de sintomas de algumas DC, o paciente jovem constrói um conceito distorcido sobre a doença e demonstra-se menos preocupado e desor- ganizado com os medicamentos, fazendo uso de forma incorreta. ${ }^{2}$ Apesar do jovem parecer ter melhor capacidade para compreender as orientações dos profissionais de saúde, ele dá menos importância ao tratamento. ${ }^{19}$

No caso do estado civil, não houve diferença significativa ( $p=0.288$ ) entre os casados, viúvos, divorciados e solteiros quando comparados ao uso correto dos medicamentos, cometeram erros ao tomar os medicamentos prescritos.

Segundo a literatura, a DC poderá acarretar limitações no estilo de vida dos pacientes, tornando-os tristes, insatisfeitos e depressivos. Assim sendo, o envolvimento de um membro da família no tratamento da DC poderia facilitar a adesão do paciente à terapia medicamentosa. ${ }^{3}$

Observa-se que a avaliação da adesão ao tratamento medicamentoso através da abordagem quantitativa pode ter sido restringida pelas especificidades das respostas quanto ao modo de uso dos medicamentos. Sugere-se o desenvolvimento de pesquisas com delineamento qualitativo.

\section{CONSIDERACÕES FINAIS}

O modo de uso dos medicamentos prescritos foi avaliado como inadequado o que poderá desencadear efeitos indesejáveis ou prejudiciais à saúde dos portadores de DCNT. Assim, sugere-se às EqSF analisar as dificuldades encontradas e propor estratégias mais efetivas de incentivo à adesão ao tratamento. No entanto, isso não é o suficiente. Faz-se necessário o envolvimento entre a equipe de saúde e os familiares, no cuidado aos indivíduos portadores de DCNT, visando a manutenção sistemática da terapia medicamentosa.

\section{REFERÊNCIAS}

1. Brasil. Ministério da Saúde. Secretaria de Vigilância em Saúde. Departamento de Análise de Situação de Saúde. Plano de ações estratégicas para o enfrentamento das doenças crônicas não transmissíveis (DCNT) no Brasil 20112022. Brasília: Ministério da Saúde, 2011.160 p.: il. - (Série B. Textos Básicos de Saúde).

2. Silva AP, Avelino FVSD, Sousa CLA, Valle ARMC, Figueiredo MLF. Fatores associados à não adesão ao tratamento da hipertensão arterial sistêmica: uma revisão integrativa. Revista de Pesquisa: Cuidado é fundamental Online 2016; 8(1):4047-4055. doi: 10.18066/revunivap.v20i35.182.

3. Barreto MS, Cremonese IZ, Janeiro V, Matsuda LM, Marcon SS. Prevalência de não adesão à farmacoterapia anti-hipertensiva e fatores associados. Revista Brasileira de Enfermagem 2015;68(1):60-7. doi: 10.1590/00347167.2015680109p.

4. Mastroianni PC, Lucchetta RC, Sarra JR, Galduróz JCF. Estoque doméstico e uso de medicamentos em uma população cadastrada na estratégia saúde da família no Brasil. Revista Panamericana Salud Publica 2011;29(5):35864. doi: 10.1590/S1020-49892011000500009.

5. Cardoso CK, Malheiros RT, Torres OM, Silveira MPT. Atenção farmacêutica domiciliar: série de casos de usuários do programa práticas integradas em saúde coletiva. Revista Ciências Farmacêuticas Básica e Aplicada 2013;34(2):263- 
268. doi: http://serv-bib.fcfar.unesp.br/seer/index.php/ Cien_Farm/article/viewPDFInterstitial/2431/1411.

6. Fabrício SCC, Wehbe G, Nassur FB, Andrade Jl. Assistência domiciliar: a experiência de um hospital privado do interior paulista. Revista Latino-americana Enfermagem 2004; 12(5):721-6. doi: 10.1590/S0104-11692004000500004.

7. Instituto Brasileiro de Geografia e Estatística. Censo 2010. http://www.ibge.gov.br/http://cidades.ibge.gov.br/xtras/ perfil.php?codmun $=315340$

8. R Core Team (2016). R: A language and environment for statistical computing. R Foundation for Statistical Computing. https://www.r-project.org/

9. Organização Mundial de Saúde (OMS). Adherence to LongTerm Therapies: evidence for action[Internet]. Geneva. OMS. 2003.199p.

10. Delgado $A B$, Lima ML. Contributo para a validação concorrente de uma medida de adesão aos tratamentos. Psic Saúde Doenças 2001;2(2):81-100.

11. MacLaughlin EJ, Raehl CL, Treadway AK, Sterling $T L$, Zoller DP, Bond CA. Assessing medication adherence in the elderly: which tools to use in clinical practice? Drugs Aging 2005;22(3):231-55.

12. Freitas JGA, Nielson SEO, Porto CC. Adesão ao tratamento farmacológico em idosos hipertensos: uma revisão integrativa da literatura. Rev Soc Bras Clin Med 2015;13(1):75-84.

13. Dourado CS, Macedo-Costa KNF, Oliveira JS, Leadebal ODCP, Silva GRF. Adesão ao tratamento de idosos com hipertensão em uma unidade básica de saúde de Joao Pessoa, Estado da Paraíba. Acta Sci Health Sci 2011;33(1):9-
17. doi: 10.4025 /actascihealthsci.v33i1.7708.

14. Santa-Helena ET, Nemes MIB, Neto JE. Fatores associados à não adesão ao tratamento com anti-hipertensivos em pessoas atendidas em unidades de saúde da família. Cad Saúde Pública 2010;26(12):2389-98. doi: 10.1590/S0102$311 \times 2010001200017$.

15. Ferreira RA, Barreto SM, Giatti L. Hipertensão arterial referida e utilização de medicamentos de uso contínuo no Brasil; um estudo de base populacional. Cad Saúde Pública 2014;30(4): 815-26

16. Busnello R, Melchior R, Faccin C, Vettori D, Petter J, Moreira LB, Fuchs FD. Características Associadas ao Abandono do Acompanhamento de Pacientes Hipertensos Atendidos em um Ambulatório de Referência. Arq Bras Cardiol 2001;76:349-51. doi: http://publicacoes.cardiol.br/ abc/2001/7605/7605001.pdf.

17. Carvalho ALM, Leopoldino RWD, Silva JEG, Cunha CP. Adesão ao tratamento medicamentoso em usuários cadastrados no Programa Hiperdia no município de Teresina (PI). Ciência \& Saúde Coletiva 2012;17(7):1885-1892. doi: 10.1590/S1413-81232012000700028.

18. Rocha $\mathrm{CH}$, Oliveira $\mathrm{SA}$, Ferreira $\mathrm{C}$, Faggiani $\mathrm{FT}$, Scroeter G, Souza AC, DeCarli G, Morrone FB, et al. Adesão a prescrição médica em idosos de Porto Alegre, RS. Ciênc. Saúde Coletiva 2008;13(3):703-10. doi: 10.1590/S141381232008000700020 .

19. Kungäs $H$, Lahdenperä $T$. Compliance of patients with hypertension and associated factors. J Adv Nurs 1999;29(4):832-9. doi: 10.1046/j.1365-2648.1999.00962.x.

Como citar: CAIXETA, Nádia Camila Rodrigues Costa et al. Modo de uso dos medicamentos de portadores de doencas crônicas acompanhados pelas equipes da estratégia de saúde da família de um município mineiro. Cinergis, Santa Cruz do Sul, v. 18, n. 1, out. 2016. ISSN 2177-4005. Disponivel em: <https://online.unisc.br/seer/index.php/cinergis/article/view/8140>. Acesso em: 11 out. 2016. doi:http://dx.doi.org/10.17058/cinergis.v18i1.8140. 